Honam Mathematical J. 33 (2011), No. 4, pp. 629-633

http://dx.doi.org/10.5831/HMJ.2011.33.4.629

\title{
TAUTNESS AND HYPERBOLICITY OF MODEL DOMAINS
}

\author{
Sung-HeE PARK
}

\begin{abstract}
In this paper we study some properties related to hyperbolicity and tautness of model domains.
\end{abstract}

\section{Introduction}

In several complex variable, model domains have been studying as prototypes of the general situation. In particular, in [6], J. Yu has proved some properties on model domain related to tautness and completeness(w.r.t invariant distances) in studying local geometrical structures of finite types domains in $\mathbb{C}^{n}$.

The goal of this note is to prove the following (notations and terminology are standard and will be recalled in the next section):

Theorem 1.1. Let $P: \mathbb{C}^{n} \rightarrow[-\infty, \infty)$ be upper semicontinuous and put

$$
G=G_{P}:=\left\{(z, w) \in \mathbb{C}^{n} \times \mathbb{C}: u(z, w):=\operatorname{Re} w+P(z)<0\right\} .
$$

Then we have that:

(a) if $G$ is pseuodoconvex, then $P$ is plurisubharmonic in $\mathbb{C}^{n}$;

(b) if $G$ is Brody hyperbolic, then $P$ is real-valued on $\mathbb{C}^{n}$;

(c) if $G$ is Kobayashi hyperbolic, then $P$ is locally bounded on $\mathbb{C}^{n}$;

(d) if $G$ is taut, then $P$ is a real-valued continuous plurisubharmonic function on $\mathbb{C}^{n}$.

Notice that the function $P\left(z_{1}, z_{2}\right):=\left|z_{1} z_{2}\right|^{2}$ is a real-valued continuous plurisubharmonic function on $\mathbb{C}^{2}$, but the domain $G_{P}=\left\{\left(z_{1}, z_{2}, w\right) \in\right.$ $\left.\mathbb{C}^{3}: \operatorname{Re} w+P\left(z_{1}, z_{2}\right)<0\right\}$ is not Brody hyperbolic(so not Kobayashi hyperbolic and not taut).

Received October 31, 2011. Accepted November 16, 2011.

2000 Mathematics Subject Classification. 32F45.

Key words and phrases. hyperbolic, taut, model domain. 


\section{Preliminaries}

Before we verify the theorem 1.1, let us recall some necessary notations.

For domains $G \subset \mathbb{C}^{n}, S \subset \mathbb{C}^{m}$, let us denote by $\mathcal{O}(G, S)$ the set of all holomorphic maps from $G$ to $S, \mathcal{O}(G):=\mathcal{O}(G, \mathbb{C})$.

Denote by $p$ the Poincaré distance on the unit disk $E \subset \mathbb{C}$, which, for two points $\lambda, \zeta \in E$, is given by the formula $p(\lambda, \zeta):=\tanh ^{-1}(\mid \lambda-$ $\zeta|/| 1-\bar{\lambda} \zeta \mid)$. The Kobayashi pseudodistance $k_{G}$ can be defined as the largest pseudodistance not exceeding $\tilde{k}_{G}$, where

$$
\tilde{k}_{G}(a, z):=\inf \{p(0, \lambda): \exists \varphi \in \mathcal{O}(E, G), \varphi(0)=a, \varphi(\lambda)=z\}, a, z \in G \text {. }
$$

Note that the function $k_{G}$ is positive definite, symmetric and satisfies the triangle inequality. The Kobayashi pseudodistnace has the distancedecrasing property in the following sense:

$$
d_{S}(f(a), f(z)) \leq d_{G}(a, z), \quad f \in \mathcal{O}(G, S), \quad a, z \in G .
$$

In particular, the Kobayashi pseudodistance is preserved by biholomorphic maps and the Kobayashi pseudodistance of the unit disc coincides with the Poincaré distance. We say that $G$ is Kobayashi hyperbolic if its Kobayashi pseudodistance is actually a distance, that is, $k_{G}(a, z)>0$ for $a, z \in G, a \neq z$. Another well-known notion of complex hyperbolicity is Brody hyperbolic, which means there are no non-constant holomorphic maps from $\mathbb{C}$ to $G$. It is well-known that Kobayashi hyperbolicity implies Brody hyperbolicity.

Proposition 2.1. ([2]) For every domain $G \subset \mathbb{C}^{n}$, the Kobayashi pseudodistance is continuous on $G \times G$, and the function $\tilde{k}_{G}$ is upper semicontinuous on $G \times G$.

For more details about general properties, refer to [2].

Denote by $\mathcal{P S H}(G)$ the set of all plurisubharmonic functions on $G$. A domain $G \subset \mathbb{C}^{n}$ is called pseuodoconvex if $-\log \operatorname{dist}(\cdot, \partial G) \in \mathcal{P} \mathcal{S H}(G)$.

Proposition 2.2. ([1]) If $D \subset \mathbb{C}^{n}$ and $\Omega \subset \mathbb{C}^{m}$ are pseudoconvex, and $f \in \mathcal{O}\left(D, \mathbb{C}^{m}\right)$, then $f^{-1}(\Omega)$ is pseudoconvex.

For the sake of more information for the pseudoconvexity we refer to [1], [3].

By $\mathcal{C}^{\uparrow}(G)$ we denote the set of all upper semicontinuous function $u: G \longrightarrow[-\infty,+\infty)$. 
Proposition 2.3. ([3]) A Harotgs-Laurent domain

$$
\Sigma_{u, v}(G):=\left\{(z, \lambda) \in G \times \mathbb{C}: e^{v(z)}<|\lambda|<e^{-u(z)}\right\},
$$

where $G \subset \mathbb{C}^{n}$ is a domain and $u, v \in \mathcal{C}^{\uparrow}(G)$ with $u+v<0$ on $G$, is pseudoconvex if and only if its base $G$ is pseudoconvex and $u, v \in$ $\mathcal{P S H}(G)$.

Let us recall the concept of taut domains introduced by Wu. A domain $G \subset \mathbb{C}^{n}$ is said to be taut if $\mathcal{O}(E, G)$ is a normal family, which means that for any sequence $\left(f_{\nu}\right)_{\nu \geq 1} \subset \mathcal{O}(E, G)$ there is a subsequence $\left(f_{\nu_{j}}\right)_{j \geq 1}$ which is either normally convergent in $\mathcal{O}(E, G)$, i.e. it converges uniformly on compact subsets to a map $f$ in $\mathcal{O}(E, G)$ (shortly $f_{\nu_{j}} \stackrel{j \rightarrow \infty}{\Longrightarrow}$ $f)$, or compactly divergent, i.e. for any compact subsets $K \subset E, L \subset G$, the set $f_{\nu_{j}}(K) \cap L$ is empty for all sufficiently large $j$. It is known that taut domains in $\mathbb{C}^{n}$ are always pseudoconvex and Kobayashi hyperbolic, but these domains cannot be taut.

\section{The Proof of Theorem 1.1}

Let $P \in \mathcal{C}^{\uparrow}\left(\mathbb{C}^{n}\right)$ and let $G=G_{P}$ be as in Theorem 1.1.

(a) Consider the Hartogs-Laurent domain $\Sigma \equiv \Sigma_{P,-\infty}\left(\mathbb{C}^{n}\right)$ over $\mathbb{C}^{n}$. We define a holomorphic function $F: \mathbb{C}^{n} \times(\mathbb{C} \backslash\{0\}) \longrightarrow \mathbb{C}^{n+1}$ by $F(z, w):=(z, \log w)$ for $(z, w) \in \mathbb{C}^{n} \times(\mathbb{C} \backslash\{0\})$, where $\log w:=\ln |w|+$ $i \operatorname{Arg} w$. Then it is easy to show that $F^{-1}(G)=\Sigma$, and so it follows from Proposition 2.2 that $\Sigma$ is pseudoconvex. Moreover, by Proposition 2.3, we have $P \in \mathcal{P S H}\left(\mathbb{C}^{n}\right)$.

(b) If $P\left(z_{0}\right)=-\infty$ for some $z_{0} \in \mathbb{C}^{n}$, then the map $\varphi:=\left(z_{0}, \cdot\right)$ is nonconstant holomorphic from $\mathbb{C}$ into $G$, so the domain $G$ is not Brody hyperbolic.

(c) Assume that $G$ is Kobayashi hyperbolic, but the function $P$ is not locally bounded on $\mathbb{C}^{n}$. Then $P$ is real-valued on $\mathbb{C}^{n}$ and there exists a sequence $\left(z_{\nu}\right)_{\nu \geq 0} \subset \mathbb{C}^{n}$ such that

$$
\begin{gathered}
z_{\nu} \longrightarrow z_{0} \text { as } \nu \rightarrow \infty, \\
-1>P\left(z_{\nu}\right) \longrightarrow-\infty \text { as } \nu \rightarrow \infty .
\end{gathered}
$$

In particular, without loss of generality, we may assume that $-\infty<$ $P\left(z_{0}\right)<0$. Indeed, if $\tilde{P}:=P-P\left(z_{0}\right)-1$ and $\tilde{G}:=\left\{(z, w) \in \mathbb{C}^{n} \times \mathbb{C}:\right.$ $\operatorname{Re} w+\tilde{P}(z)<0\}$, then the map $\psi$, defined by $\psi(z, w):=(z, w+$ $\left.P\left(z_{0}\right)+1\right)$ for $(z, w) \in \mathbb{C}^{n} \times \mathbb{C}$, is biholomorphic of $G$ with $\tilde{G}$. But since the Kobayashi hyperbolicity of domains is a biholomorphic invariant 
property, $G$ is Kobayashi hyperbolic iff so is $\tilde{G}$. In particular, $\tilde{P}$ is not locally bounded at the point $z_{0}$ and $\tilde{P}\left(z_{0}\right)<0$.

For any $\nu \geq 1$ we define a map $\varphi_{\nu}: E \rightarrow \mathbb{C}^{n+1}$ by

$$
\varphi_{\nu}(\lambda):=\left(z_{\nu},-P\left(z_{\nu}\right) \lambda\right), \quad \lambda \in E .
$$

Observe that

$$
\begin{aligned}
u\left(\varphi_{\nu}(\lambda)\right) & =\operatorname{Re}\left(-P\left(z_{\nu}\right) \lambda\right)+P\left(z_{\nu}\right) \\
& =-P\left(z_{\nu}\right) \operatorname{Re} \lambda+P\left(z_{\nu}\right)<-P\left(z_{\nu}\right)+P\left(z_{\nu}\right)=0
\end{aligned}
$$

for any $\lambda \in E$ and any $\nu \geq 1$. From which we obtain that $\left(\varphi_{\nu}\right)_{\nu \geq 1} \subset$ $\mathcal{O}(E, G)$ with $\varphi_{\nu}(0)=\left(z_{\nu}, 0\right)$. Note that $\left(1 / P\left(z_{\nu}\right)\right)_{\nu \geq 1} \subset E$ and also $\varphi_{\nu}\left(1 / P\left(z_{\nu}\right)\right)=\left(z_{\nu},-1\right)$. But since $P\left(z_{0}\right)-1<P\left(z_{0}\right)<0$, it is clear that $\left(z_{0}, 0\right),\left(z_{0},-1\right) \in G$ and hence, by the continuity and the decreasing property of the Kobayashi pseudodistance, we have

$$
\begin{aligned}
0 & \leq k_{G}\left(\left(z_{0}, 0\right),\left(z_{0},-1\right)\right) \\
& =\lim _{\nu \rightarrow \infty} k_{G}\left(\left(z_{\nu}, 0\right),\left(z_{\nu},-1\right)\right) \\
& =\lim _{\nu \rightarrow \infty} k_{G}\left(\varphi_{\nu}(0), \varphi_{\nu}\left(\frac{1}{P\left(z_{\nu}\right)}\right)\right) \leq \lim _{\nu \rightarrow \infty} k_{E}\left(0, \frac{1}{P\left(z_{\nu}\right)}\right)=0 .
\end{aligned}
$$

But, this is a contradiction to the assumption that $G$ is Kobayashi hyperbolic.

(d) Suppose that $P$ is not continuous at a point $z_{0} \in \mathbb{C}^{n}$. Since $P \in \mathcal{C}^{\uparrow}\left(\mathbb{C}^{n}, \mathbb{R}\right)$ (cf. (a)), we can take a constant $A \in \mathbb{R}$ and a sequence $\left(z_{\nu}\right)_{\nu \geq 1} \subset \mathbb{C}^{n}$ such that $\lim _{\nu \rightarrow \infty} z_{\nu}=z_{0}$ and $P\left(z_{\nu}\right)<A<P\left(z_{0}\right)<A+1$ for any $\nu \geq 1$. For any $\nu \geq 1$ we define a map $\varphi_{\nu}: E \rightarrow \mathbb{C}^{n+1}$ by

$$
\varphi_{\nu}(\lambda):=\left(z_{\nu},-A+\frac{\lambda-1}{\lambda+1}\right), \quad \lambda \in E .
$$

Observe that

$$
\begin{aligned}
u\left(\varphi_{\nu}(\lambda)\right) & =\operatorname{Re}\left(-A+\frac{\lambda-1}{\lambda+1}\right)+P\left(z_{\nu}\right) \\
& =-A+\frac{|\lambda|^{2}-1}{|\lambda+1|^{2}}+P\left(z_{\nu}\right)<-A+P\left(z_{\nu}\right)<0
\end{aligned}
$$

for any $\lambda \in E$ and any $\nu \geq 1$, which implies that $\left(\varphi_{\nu}\right)_{\nu \geq 1} \subset \mathcal{O}(E, G)$. Moreover,

$$
\varphi_{\nu}(0)=\left(z_{\nu},-A-1\right) \stackrel{\nu \rightarrow \infty}{\longrightarrow}\left(z_{0},-A-1\right) \in G .
$$


But since $G$ is taut, the last property gives us that the sequence $\left(\varphi_{\nu}\right)_{\nu \geq 1}$ does not diverge compactly on $E$; and also

$$
\varphi_{\nu}(\lambda) \stackrel{\nu \rightarrow \infty}{\Longrightarrow} \varphi_{0}(\lambda):=\left(z_{0},-A+\frac{\lambda-1}{\lambda+1}\right) \in \mathcal{O}(E, G)
$$

However,

$$
u\left(\varphi_{0}(\lambda)\right)=-A+\frac{|\lambda|^{2}-1}{|\lambda+1|^{2}}+P\left(z_{0}\right) \stackrel{\mathbb{R} \ni \lambda \rightarrow 1}{\longrightarrow} P\left(z_{0}\right)-A>0 ;
$$

moreover, $\varphi_{0}(\lambda) \notin G$ for any $\lambda \in E \cap \mathbb{R}$ with

$$
\frac{1-C}{1+C}<\lambda<1, \quad C:=P\left(z_{0}\right)-A \in(0,1) .
$$

This contradicts to the fact that $\varphi_{0}(E) \subset G$.

\section{References}

[1] P. Jakóbczak \& M. Jarnicki, Lectures on holomorphic functions of several complex variables, PS File at 'http://www.im.uj.edu.pl/ jarnicki/mjp.htm', 2001.

[2] M. Jarnicki \& P. Pflug, Invariant Distances and Metrics in Complex Analysis, de Gruyter Expositions in Mathematics 9, Walter de Gruyter 1993.

[3] M. Jarnicki \& P. Pflug, First Steps in Several Complex Variables: Reinhardt Domains, European Mathematical Society Publishing House, 2008.

[4] M. Jarnicki \& P. Pflug, Invariant distances and metrics in complex analysisrevisited, Dissertationes Math. 430 (2005), 1-192.

[5] S.-H. Park, On Hyperbolicity and Tautness of Certain Hartogs Type Domains, Rocky Mountain J. Math., 37 (3) (2007), 959-985.

[6] J. Yu, Geometric analysis on weakly pseudoconvex domains, Dissertation, Washington University, 1993.

Sung-Hee Park

Department of Mathematics Education, Jeonju University, Jeonju 560-759, Korea.

E-mail:wshpark@jj.ac.kr 